\title{
ASSEMBLING SPIRAL GALAXIES
}

\author{
R.C. KENNICUTT \\ Steward Observatory \\ University of Arizona \\ Tucson, AZ 85721 USA
}

\section{Introduction}

Nearby spiral galaxies offer vital clues to some of the most fundamental questions about galaxy formation and evolution: What is the star formation history of the universe, past and future? When did disks form, during the final stages of a single primeval collapse, or as a continuous or episodic process? What is the evolutionary nature of the Hubble sequence, and what are the physical mechanisms that dictate the present-day Hubble type of a galaxy? Was Hubble type imprinted at birth, or can it be deterined or at least modified by infall, mergers, or secular dynamical evolution within the galaxy? These issues are not specific to spirals, of course, and much of this conference will address just these questions in a broader context. However present-day spirals offer unique advantages for studying these problems; they exhibit a broad range of dynamical and evolutionary properties, and the dynamical fragility of disks makes them excellent seismometers of galaxy interaction and merger rates at recent epochs.

\section{Stellar Birthrate Histories of Disks}

The only system for which detailed information is available is the Galactic disk (solar neighborhood). Several stellar age indicators are available, including isochrone fitting of stars near the main sequence turnoff, chromospheric dating of late-type stars, and cooling ages of white dwarfs. Although some details remain uncertain, such as the detailed time dependence of the stellar birthrate, the role of starbursts, and the age of the oldest disk stars, the observations are consistent with a roughly constant star formation rate (SFR) over the history of the disk, with the long-term SFR not varying by more than factors of 2-3. A convenient way to parameterize this history is 
in terms of the ratio of the current disk SFR to the average past rate, which I denote as $b$ following Scalo (1986). The observations of the Galactic disk are then consistent with $b \simeq 1 \pm 0.5$ (Scalo 1986; Noh \& Scalo 1990).

Several methods have been applied to measure $b$ for large samples of spirals, including modelling of integrated colors in the visible and ultraviolet, and use of integrated $\mathrm{H} \alpha$ emission-line fluxes to measure the SFRs directly (see Kennicutt 1992 for references). Kennicutt et al. (1994) have used $\mathrm{H} \alpha$ and broadband photometry of $210 \mathrm{Sa}$-Irr galaxies to estimate $b$ for the spiral disks, corrected for the effects of bulge contamination. The star formation history shows a strong systematic dependence on type, with $b \sim 1$ in the disks of late-type galaxies (Sbc and later), decreasing to $b<0.05$ in Sa disks. This means that the pronounced changes in the integrated spectra and stellar populations of spirals along the Hubble sequence are due overwhelmingly to changes in the disk star formation histories, with changes to the bulge/disk ratio being much less important. For example between types $\mathrm{Sa}$ and $\mathrm{Sc}$ the fractional contribution of young stars to the integrated disk population changes by at least a factor of 20 , while the disk luminosity fraction changes by only a factor of two (Kennicutt et al. 1994). Bulges may play a pivotal role in causing these changes to disk populations, but even if the bulges were invisible there would be a strong trend in integrated spectra with spiral type.

The radial dependence of the star formation history within disks can provide important clues to the physical origin of the type-dependent trends. For example most formation models predict a pronounced "inside-out" evolution due to the higher densities and shorter dynamical times at small radii (e.g., Larson 1972). The advent of wide-field CCD and infrared array cameras now makes it possible to address this problem quantitatively. De Jong (1995) has used $B V R I H K$ imaging of 86 face-on spirals to study the radial color distributions. The spirals show a trend toward bluer colors with later type as expected, but most of the disks also show significant color gradients, with bluer colors at larger radii. Similar color gradients have been observed by Terndrop et al. (1994) and Peletier et al. (1995).

Several effects can produce the color gradients, including a radial change in the star formation history, a reddening gradient, or an abundance gradient. Models by de Jong suggest that all three effects are important, and this makes it difficult to accurately measure the magnitude of the variation in $b$. However an independent study by Ryder \& Dopita (1994) offers strong evidence for radial birthrate gradients, based on a comparison of $\mathrm{H} \alpha$ and $I$-band scale lengths for 30 southern spirals. They find that the young disks $(\mathrm{H} \alpha)$ are considerably more extended, typically by $\sim 50 \%$ as compared to $I$. Taken together these results suggest that $b$ increases by factors of up to a few over the radial extent of the star forming disks. 


\section{The Conventional Model: Closed-Box Evolution}

The birthrate histories implied by these data are usually interpreted in the context of a passive closed-box model, in which the stellar birthrate is assumed to decline slowly (usually exponentially) with time. This implicitly assumes that most disks formed at early epochs, and that the differences in subsequent evolution along the Hubble sequence are due to physical differences in the properties of the proto-disks (e.g., Sandage 1986).

The simplest version of this picture treats disks as systems with identical ages and IMFs, varying only in the e-folding time of the SFR. Such models have been used to synthesize the colors, $\mathrm{H} \alpha$ emission, and integrated spectra of nearby galaxies. The model is remarkably successful at reproducing the spectra of galaxies over a wavelength range extending from the ionizing UV to the near-infrared (e.g., Kennicutt 1983; Buat et al. 1987; Bruzual \& Charlot 1993). The same model predicts a relatively modest evolution in the photometric properties of most $L^{*}$ spirals with lookback time, which appears consistent with faint galaxy surveys and Mg II absorber statistics.

Given these successes why should one be dissatisfied with the closedbox picture at all? Perhaps the most disconcerting problem is the lack of any physical basis for why the disk star formation histories should vary so strongly with parent galaxy type. One normally thinks of the SFR being driven by the local gas surface density (e.g., Kennicutt 1989), but if density controlled the evolutionary time scale we would expect to observe very strong radial trends in $b$ and only weak trends with galaxy type, whereas the opposite trends are observed. Closed-box models also have well-known difficulties in reproducing the observed metal abundance distributions and radial abundance gradients in disks (e.g., Zaritsky 1995).

Hence we are forced to conclude that either the closed-box assumption is invalid (see next section), or that a physical mechanism other than the Schmidt gas density law is responsible for the strong changes in star formation timescale along the Hubble sequence. Early-type spirals possess large central bulges, and the bulges might influence the disk evolution in several ways: by increasing the angular rotation speeds in the inner disks and stimuating star formation; by raising the threshold density for star formation at later epochs; or by inducing strong spiral density waves at early times. Alternatively the evolutionary differences may be connected to the systematic trend toward higher galaxy mass toward early types. Or it is possible that some spirals evolve dynamically from late to early types, for instance by forming bulges by disk heating after the formation of a massive bar (Pfenniger \& Norman 1990; Friedli \& Martinet 1993). Some combination of these mechanisms might account for the evolutionary changes of disks along the Hubble sequence without having to abandon the closed-system assumption. 


\section{Revisionist Interpretations: Infall, Captures, Mergers}

In a hierarchical model of galaxy formation one would not expect galaxies to evolve as closed systems at all, and indeed it is conceivable that the present-day Hubble type of a spiral could be largely dictated by its disk accretion or merger history. Here we discuss models for accretion-driven spiral galaxy evolution, including infall models, in which the accretion of gas is continuous, and merger models, in which disks grow by the capture of discrete clumps of gas and stars.

\subsection{INFALL-DOMINATED EVOLUTION?}

Infall can solve several of the problems alluded to earlier, extending the star formation timescales of late-type galaxies and more readily explaining the abundance distributions and gradients in disks. However recent observations appear to limit the amount of infall to rates which are well below the present-day SFRs in massive spirals. HI measurements reveal no large reservoirs of cold gas (Bothun 1985), and the inflow rate from high-velocity clouds in the Galaxy are several times smaller than that needed to sustain the current SFR (Mirabel \& Morris 1990). Perhaps the strongest limit comes from the soft X-ray luminosities of spirals (White \& Frenk 1991). ROSAT observations of nearby spirals yield diffuse X-ray luminosities that are several times smaller than the values expected from a cooling flow if the infall rate were equal to the gas depletion rate from star formation. Hence an extreme spherical infall model appears to be ruled out. However some fraction of the star formation could be fueled by infall or by a radial inflow of $\mathrm{HI}$ from the outer disk.

\subsection{MERGER-DOMINATED EVOLUTION?}

These constraints can be avoided if the accretion of gas takes place in discrete events, such as by the merger of a spiral with a companion or satellite galaxy. Indeed such hierarchical growth is a fundamental consequence of many galaxy formation models (e.g., White \& Frenk 1991; Kauffmann \& White 1993). A serious problem with this picture however is the observed thinness and dynamical coldness of disks. Numerical simulations show that much of the dynamical friction loss in a merger is converted into thermal energy of the disk (Quinn et al. 1993). Tóth \& Ostriker (1992) have estimated that no more than $4 \%$ of the mass of the disk within the solar radius could have been accreted in the last 5 Gyr. These results appear to rule out recent mergers of stellar systems (e.g., satellite galaxies) as the predominant mechanism for assembling disks, but these limits may not apply to the accretion of largely gaseous (dissipative) material. 


\subsection{OBSERVATIONAL EVIDENCE FOR ACCRETION EVENTS}

A very exciting development in this field has been the discovery of numerous examples of disks with distinct kinematic subsystems in stars and/or gas, which must represent the products of at least two discrete accretion events. These observations offer direct evidence that captures of galaxies or gas clouds play some role in disk evolution, though as discussed later the role of such mergers at recent epochs is probably a minor one.

Polar Ring Galaxies: Polar ring galaxies represent the best known and perhaps the best studied examples of multiple kinematic subsystems in spirals. Whitmore et al. (1990) catalog the $\sim 70$ known systems and provide a bibliography to the extensive literature on these objects. They used these data to estimate that roughly $5 \%$ of S0 galaxies are estimated to have had a polar ring over their lifetime.

Star-Star Counterrotation: Multiple kinematic components in a coplanar disk are more difficult to detect, but two spectacular instances of such disks are now known. The disk of the edge-on S0 galaxy NGC 4550 is comprised of two counterrotating components with nearly identical masses and scale lengths (Rubin et al. 1992; Rix et al. 1992). Both disks are cold with dispersions of 45 and $60 \mathrm{~km} \mathrm{~s}^{-1}$. In the normal Sb galaxy NGC $721720-30 \%$ of the disk stars are in a retrograde cold disk (Merrifield \& Kuijken 1994). Counterrotating stellar subsystems are seen frequently in elliptical galaxies, and are readily explained as resulting from stellar mergers or gas infall. NGC 4550 and NGC 7217 show that it is possible to form a stable counterrotating disk without violating the disk heating constraint (Sellwood \& Merritt 1993). Such systems may form from two distinct generations of disk accretion and star formation, or by an extended period of infall in which the angular momentum of the accreted material undergoes a rapid change (Quinn \& Binney 1992; Merrifield \& Kuijken 1994).

Star-Gas Counterrotation: The most frequently observed instances of counterrotation involve gas disks counterrotating with respect to the stars. A compilation of S0 galaxies by Bertola et al. (1992) lists 9 clear examples of gas-stellar counterrotation. In most instances the gas is confined to the nuclear regions, and the mass involved is small, of order $10^{6} M_{\odot}$ or less. However in at least two galaxies, NGC 4526 (Bettoni et al. 1991) and NGC 3626 (Cirri et al. 1995) the counterrotation extends over the entire disk and the gas masses are of order $10^{8}-10^{9} M_{\odot}$. These systems most likely originate from the capture of a gas-rich companion in a retrograde orbit.

Gas-Gas Counterrotation: NGC 4826 (M64) represents a rare example of a galaxy in which the rotation of the gas disk reverses itself, at a radius of $\sim 1 \mathrm{kpc}$, coincident with its prominent dust lane (Braun et al. 1992). The 
inner system, probably the remnant of the original gas disk, corotates with the stars. Outside of $1 \mathrm{kpc}$ the gas is in retrograde rotation (Rubin 1994; Rix et al. 1995). The gas motions in the transition region are complex, with much of the gas moving at less than the circular velocity. However the stellar disk exhibits a normal exponential profile, a dynamically cold disk, and no discernable counterrotation (Walterbos et al. 1994; Rix et al. 1995). As with the systems described above NGC 4826 is most likely the result of the capture of a gas-rich companion. The absence of retrograde stars and the cold stellar disk appear to rule out a significant mass in stars in the captured object, but the presence of strong [OIII], [NII], and [SII] forbidden lines in the outer disk suggest a significant degree of chemical enrichment (and hence star formation) in the captured object.

Infalling Gas Clouds Evidence for infalling gas in nearby spirals comes from a variety of sources. High-velocity HI clouds with masses of up to $2 \times 10^{8} M_{\odot}$ have been detected in M101, NGC 628, and NGC 6946 (Kamphuis 1993). High-resolution HI observations also reveal large-scale kinematic structures in the form of massive arms and arcs of gas that may still be settling into disks, as in IC 10 (Hodge et al. 1994). See Sancisi's review in this volume for a more extensive discussion of this subject. Infalling ionized gas has been observed in the early-type spirals NGC 4258 (Rubin \& Graham 1990) and NGC 4826 (Rubin 1994). Dynamical modelling of mergers suggests that a steady infall of gas can persist for billions of years after a merger (Hibbard \& Mihos 1995).

\subsection{IMPLICATIONS FOR DISK FORMATION AND EVOLUTION}

The many examples described above indicate that episodic accretion does play a significant role in disk evolution, and that significant accretion can take place without disrupting or overheating the disks. However these types of subsystems probably comprise no more than a few percent of the presentday mass of disks. Rix and Kuijken (private communications) have completed surveys for stellar counterrotation of the type seen in NGC 4550 for $\sim 30-40 \mathrm{~S} 0$ and spiral galaxies and have failed to detect counterrotation in any, implying upper limits of a few percent on the fraction of retrograde stars. Similar fractions were derived from polar rings by Whitmore et al. (1990). Counterrotating gas disks may be more common, but the masses involved are rarely more than a few percent of the total disk mass. These numbers are reminiscent of the limits based on disk heating derived for the Galactic disk by Tóth \& Ostriker (1992).

This result may have important implications for current models of galaxy formation. In an $\Omega=1 \mathrm{CDM}$ model most $L^{*}$ galaxies experience major halo mergers over the past few Gyr (Kauffmann \& White 1993), and even if only 
a fraction of these events lead to stellar/gas disk mergers the predictions would appear to be in conflict with the observations described above. On the other hand in an $\Omega=0.2$ model most of the galaxy assembling occurs at early cosmological epochs, and the fraction of recent mergers, of order a few tens of percent or less, is in reasonable accord with the observations of disks. While hardly conclusive this example illustrates the importance of disk galaxies in constraining the merger rate and the nature of the galaxy formation paradigm.

I am very grateful to several colleagues for stimulating discussions and for sharing new results prior to discussion, especially Marc Balcells, Robert Braun, Roelof de Jong, John Hibbard, Konrad Kuijken, Reynier Peletier, Hans-Walter Rix, Renzo Sancisi, Peter Tamblyn, Chris Taylor, Thijs van der Hulst, Jacqueline van Gorkom, Rene Walterbos, Simon White, and Eric Wilcots. My work was supported in part by the U.S. National Science Foundation through grants AST-9019150 and AST-9421145.

\section{References}

Bertola, F., Buson, L. M., \& Zeilinger, W. W. 1992, ApJ, 401, L79

Bettoni, D., Galletta, G., \& Oosterloo, T. 1991, MNRAS, 248, 544

Bothun, G. D. 1985, AJ, 90, 1982

Braun, R., Walterbos, R. A. M., \& Kennicutt, R. C. 1992, Nature, 360, 442

Bruzual, G., \& Charlot, S. 1993, ApJ, 405, 538

Buat, V., Donas, J., \& Deharveng, J. M. 1987, A\&A, 185, 33

Cirri, R., Bettoni, D., \& Galletta, G. 1995, Nature, in press

de Jong, R. 1995, Ph.D. thesis, Univ. of Groningen

Friedli, D., \& Martinet, L. 1993, A\&A, 277, 27

Hibbard, J. E., \& Mihos, J. C. 1995, AJ, 110, 140

Hodge, P., Wilcots, E., \& Miller, B. 1994, BAAS, 26, 1436

Kamphuis, J. 1993, Ph.D. thesis, Univ. of Groningen

Kauffmann, G., \& White, S. D. M. 1993, MNRAS, 261, 921

Kennicutt, R. C. 1983, ApJ, 272, 54

Kennicutt, R. C. 1989, ApJ, 344, 685

Kennicutt, R. C. 1992, in Star Formation in Stellar Systems, eds. G. Tenorio-Tagle, M. Prieto, \& F. Sánchez (Cambridge: Cambridge Univ. Press), p191

Kennicutt, R. C., Tamblyn, P., \& Congdon, C. W. 1994, ApJ, 435, 22 (KTC)

Larson, R. B. 1972, Nature, 236, 21

Merrifield, M. R., \& Kuijken, K. 1994, ApJ, 432, 575

Mirabel, F., \& Morris, M. 1990, ApJ, 356, 130

Noh, H.-R., \& Scalo, J. M. 1990, ApJ, 352, 605

Peletier, R. F., Valentijn, E. A., Moorwood, A. F. M., Freudling, W., Knapen, J. H., \& Beckman, J. E. 1995, A\&A, in press

Pfenniger, D., \& Norman, C. 1990, ApJ, 363, 391

Quinn, P. J., Hernquist, L, \& Fullagar, D. P. 1993, ApJ, 403, 74

Quinn, T., \& Binney, J. 1992, MNRAS, 255, 729

Rix, H.-W., Franx, M., Fisher, D., \& Illingworth, G. 1992, ApJ, 400, L5

Rix, H.-W., Kennicutt, R. C., Braun, R., \& Walterbos, R. A. M. 1995, ApJ, 438, 155

Rubin, V. C. 1994, AJ, 107, 173

Rubin, V. C., \& Graham, J. A. 1990, ApJ, L5 
Rubin, V., Graham, J., \& Kenney, J. 1992, ApJ, 394, L9

Ryder, S. D., \& Dopita, M. A. 1994, ApJ, 430, 142

Sandage, A. 1986, A\&A, 161, 89

Scalo, J. M. 1986, Fund. Cos. Phys., 5, 287

Sellwood, J. A., \& Merritt, D. 1994, ApJ, 425, 530

Terndrop, D. M., Davies, R. L., Frogel, J. A., DePoy, D. L., \& Wells, L. A. 1994, ApJ, 432,518

Tóth, G., \& Ostriker, J. P. 1992, ApJ, 389, 5

Walterbos, R. A. M., Braun, R., \& Kennicutt, R. C. 1994, AJ, 107, 184

White, S. D. M., \& Frenk, C. S. 1991, ApJ, 379, 52

Whitmore, B. C., Lucas, R. A., McElroy, D. B., Steiman-Cameron, T. Y., Sackett, P. D., \& Olling, R. P. 1990, AJ, 100, 1489

Zaritsky, D. 1995, ApJ, 448, L17

\section{Discussion}

McCall: The Hubble sequence is essentially a disk sequence because in the Revised Hubble System disk properties are favoured over bulge/disk ratio. At a given Hubble stage, the bulge/disk ratio can vary systematically. Is there any evidence for a variation of the star formation rate (characterized by $b$ ) with the bulge/disk ratio at a given Hubble stage?

Kennicutt: This is difficult to test, because of the scarcity of good bulge/disk photometry for a large enough sample. I have looked a correlations of SFR with Yerkes type, which classifies on the basis of bulge/disk ratio, and the scatter is similar to that seen when the SFR is correlated with RSA or RC2 type.

Minniti: What fraction of these galaxies with counter-rotating disks are barred?

Kennicutt: Counterrotation is seen in barred and non-barred galaxies, but the number of known systems is too small to test whether bars are more or less common in counterrotating systems. For nearly edge-on systems such as NGC 4550 it is difficult to tell if a bar is present.

Athanassoula: It is possible to produce a retrograde component without invoking a merger. Namely, if a bar decayed and turned into an axisymmetric component (e.g., because of an increase in the central mass concentration) then a fraction of the orbits which were ergodic before the decay will become retrograde.

Freeman: I heard secondhand that Hernquist et al. have modelled accretion events on disk galaxies with live halos and this makes the disks more robust. Does anyone know about this?

Athanassoula: The simulations of Quinn et al. have a rigid halo. I have run a series of simulations in which the halo, as well as the disk and companion, are live. In some of these simulations the companion loses a substantial fraction of its mass while it is still in the halo. This depends on how dense the companion is. 